\title{
Monitoring Response to Neoadjuvant Chemotherapy of Primary Osteosarcoma Using Diffusion Kurtosis Magnetic Resonance Imaging: Initial Findings
}

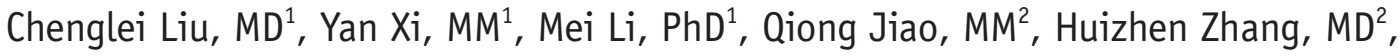 \\ Qingcheng Yang, $\mathrm{PhD}^{3}$, Weiwu Yao, $\mathrm{PhD}^{1}$ \\ Departments of ${ }^{1}$ Radiology, ${ }^{2}$ Pathology, and ${ }^{3}$ Orthopedics, Shanghai Jiao Tong University Affiliated Sixth People's Hospital, Shanghai, China
}

Objective: To determine whether diffusion kurtosis imaging (DKI) is effective in monitoring tumor response to neoadjuvant chemotherapy in patients with osteosarcoma.

Materials and Methods: Twenty-nine osteosarcoma patients (20 men and 9 women; mean age, $17.6 \pm 7.8$ years) who had undergone magnetic resonance imaging (MRI) and DKI before and after neoadjuvant chemotherapy were included. Tumor volume, apparent diffusion coefficient (ADC), mean diffusivity (MD), mean kurtosis (MK), and change ratio ( $\triangle \mathrm{X}$ ) between preand post-treatment were calculated. Based on histologic response, the patients were divided into those with good response ( $\geq 90 \%$ necrosis, $n=12$ ) and those with poor response $(<90 \%$ necrosis, $n=17)$. Several MRI parameters between the groups were compared using Student's $t$ test. The correlation between image indexes and tumor necrosis was determined using Pearson's correlation, and diagnostic performance was compared using receiver operating characteristic curves.

Results: In good responders, $\mathrm{MD}_{\text {post }} \mathrm{ADC}_{\text {post }}$ and $\mathrm{MK}_{\text {post }}$ values were significantly higher than in poor responders $(p<0.001$, $p<0.001$, and $p=0.042$, respectively). The $\triangle M D$ and $\triangle A D C$ were also significantly higher in good responders than in poor responders $\left(p<0.001\right.$ and $p=0.01$, respectively). However, no significant difference was observed in $\triangle \mathrm{MK}(p=0.092)$. $M D_{\text {post }}$ and $\triangle M D$ showed high correlations with tumor necrosis rate $\left(r=0.669\right.$ and $r=0.622$, respectively), and $\mathrm{MD}_{\text {post }}$ had higher diagnostic performance than $\mathrm{ADC}_{\text {post }}(p=0.037)$ and $\mathrm{MK}_{\text {post }}(p=0.011)$. Similarly, $\triangle \mathrm{MD}$ also showed higher diagnostic performance than $\triangle \mathrm{ADC}(p=0.033)$ and $\triangle \mathrm{MK}(p=0.037)$.

Conclusion: $M D$ is a promising biomarker for monitoring tumor response to preoperative chemotherapy in patients with osteosarcoma.

Keywords: Bone neoplasm; Functional MRI; Diffusion; Treatment

\section{INTRODUCTION}

Osteosarcoma is the most common primary malignant bone tumor in childhood and adolescence. Since neoadjuvant chemotherapy was introduced in the 1970s, patients' long-term survival rates have dramatically improved (1). Combined surgery and chemotherapy leads to long-term, disease-free survival in approximately $60 \%$ of patients with a localized extremity lesion (2). To date, more than $90 \%$ of chemotherapy-induced tumor necrosis in osteosarcoma has been considered as the most reliable predictive indicator of survival (3). However, histological

Received July 17, 2018; accepted after revision November 11, 2018.

This study was supported by Shanghai Scientific Research Plan Project (No. 16511101100, No. 16511101101), National Natural Science Foundation of China (No. 81771790), and Shanghai key discipline of medical imaging (No. 2017ZZ02005). They have no role in study design, data collection, analysis, interpretation or in writing the manuscript.

Corresponding author: Weiwu Yao, PhD, Department of Radiology, Shanghai Jiao Tong University Affiliated Sixth People's Hospital, 600 Yi shan Road, Shanghai 200233, China.

- Tel: (86) 18930177504•Fax: (86) 021-64368290•E-mail: yaoweiwuhuan@163.com

This is an Open Access article distributed under the terms of the Creative Commons Attribution Non-Commercial License (https:// creativecommons.org/licenses/by-nc/4.0) which permits unrestricted non-commercial use, distribution, and reproduction in any medium, provided the original work is properly cited. 
tumor necrosis can only be assessed postoperatively. In clinical practice, knowledge of tumor necrosis in vivo is very important to allow physicians to modify chemotherapy regimens during the course of chemotherapy and to allow surgeons to individualize surgical planning (limb-sparing or amputation); ineffective chemotherapy may potentially increase the risk of iatrogenic toxicity or formation of resistant clones (4). Patients' subjective responses and clinical examinations have proven less than satisfactory (5). Therefore, it would be desirable to have an imaging surrogate to monitor response to neoadjuvant chemotherapy prior to surgical resection.

To overcome these limitations, several modalities, including radiography, computed tomography (CT), and conventional magnetic resonance imaging (MRI), have been used to monitor tumor chemotherapy response. However, these anatomical imaging modalities are insufficient to monitor tumor necrosis due to the overlap of different morphological features resulting from chemotherapy, such as edema, granulation tissue, and fibrosis (6). Several recent studies have reported on the usefulness of dynamic MRI for the assessment of tumor necrosis after chemotherapy $(5,7)$. However, the routine use of dynamic MRI has been limited by its time-consuming nature and heavy workload for postprocessing.

Diffusion weighted imaging (DWI) is a non-invasive, functional MRI modality. Mounting evidence has shown that DWI could directly monitor tumor therapeutic response and that apparent diffusion coefficient (ADC) values were closely related to tumor necrosis (8-14). However, this imaging modality assumes that water diffusion in tissue follows Gaussian diffusion behavior. In fact, water diffusion is heterogeneous because of the presence of various barriers and compartments in the tissue microstructure, especially in bone tumors. Diffusion kurtosis imaging (DKI) offers a method of evaluating diffusional heterogeneity and structural connectivity in complex biological tissues, which may potentially augment conventional diffusion techniques for better monitoring of response to treatment. In previous studies, DKI has been used to monitor treatment response in patients with locally advanced rectal cancer and advanced nasopharyngeal carcinoma $(15,16)$. However, to our knowledge, there is no published study regarding the application of DKI for monitoring chemotherapy response in patients with osteosarcoma. Therefore, our goal was to determine whether DKI has the power to further monitor tumor necrosis in patients with osteosarcoma.

\section{MATERIALS AND METHODS}

\section{Patients}

Our prospective study was approved by the Ethics Committee of Shanghai Sixth People's Hospital (YS-2016064), and the written informed consent was obtained from all patients in this study. The inclusion criteria for patients were as follows: 1) newly pathological diagnosis of primary high-grade extremity osteosarcoma and no treatment before the MRI scan; 2) completion of neoadjuvant chemotherapy and surgery; 3) MRI scans obtained before and after the completion of neoadjuvant chemotherapy, with a time interval between the first MRI and the initiation of neoadjuvant chemotherapy of no more than 2 weeks, and a time interval between follow-up MRI and surgery of no more than 2 weeks. Thirty-two consecutive patients were prospectively registered between March 2016 and February 2017. Three patients were excluded due to serious motion artifacts after neoadjuvant chemotherapy. Thus, 29 patients (women, $\mathrm{n}=9$; men, $\mathrm{n}=20$; age range, 7-34 years; mean age, $17.6 \pm 7.8$ years) were enrolled in this study. All patients received four cycles of chemotherapy at intervals of 2-3 weeks. The chemotherapy regimen was a combination of cisplatin at a dose of $40 \mathrm{mg} / \mathrm{m}^{2}$ and doxorubicin at a dose of $25 \mathrm{mg} / \mathrm{m}^{2}$ for 3 days.

\section{MRI Protocols}

All studies were performed using a $3 \mathrm{~T}$ superconducting magnetic resonance (MR) scanner (MAGNETOM Verio, Siemens Healthineers, Erlangen, Germany) with a dedicated eight-channel body array coil. Conventional MR sequence included fat-saturated T2-weighted sequences (repetition time [TR]/echo time [TE], $4000 \mathrm{msec} / 104 \mathrm{msec}$; field of view [FOV], $380 \mathrm{~mm}$; image matrix, $384 \times$ 384; section thickness, $5 \mathrm{~mm}$ ) in the coronal, sagittal and axial planes and a coronal T1-weighed sequence (TR/TE $600 \mathrm{msec} / 20$ msec; FOV, $380 \mathrm{~mm}$; image matrix, $384 \times$ 384; section thickness, $5 \mathrm{~mm}$ ). Contrast-enhanced MRI was performed using fat-saturated T1-weighted sequences in the coronal, sagittal, and transverse planes after injection of $0.1 \mathrm{mmol} /$ $\mathrm{kg}$ diethylenetriaminepentaacetic acid (Magnevist, Bayer AG, Berlin, Germany) into the median cubital vein. The scan parameters were identical to those described above.

DKI was obtained before contrast material injection. DKI (with b values of $0,500,1000,1500,2000$, and $2500 \mathrm{sec} / \mathrm{mm}^{2}$ ) was performed in the axial plane using an echo planar sequence with fat-suppression (TR/TE, 3400 
msec/72 msec; FOV, 380 mm; image matrix, 384 x 384; section thickness, $5 \mathrm{~mm}$; flip angle, $180^{\circ}$; voxel size, 2.0 $\times 2.0 \times 5 \mathrm{~mm}^{3}$; acquisition time, 4 minutes 37 seconds). The diffusion gradients were encoded in three orthogonal directions.

\section{MR Image Analysis}

The parameter maps of both DKI and DWI were obtained using the prototype post-processing software "Body Diffusion Toolbox" created with MATLAB (Math Works, Natick, MA, USA) (17). An ADC map was calculated using a monoexponential model (with $b$ values of 0 and $1000 \mathrm{sec} /$ $\mathrm{mm}^{2}$ ) based on the following equation: $\ln (\mathrm{S})=\ln (\mathrm{S} 0)-\mathrm{b}$. $A D C$, where $S$ is the signal intensity at diffusion-weighting value $b$, and $S 0$ is signal intensity at $b=0$. The kurtosis and diffusivity maps were calculated from DKI with $b$ values of $0,500,1000,1500,2000$, and $2500 \mathrm{sec} / \mathrm{mm}^{2}$ based on the DKI equation: $\ln (S)=\ln (S 0)-b \cdot D+1 / 6 \cdot b^{2} \cdot D^{2}$ - $K$, where $S$ is the signal at $b$ value, $S 0$ is the baseline signal without diffusion gradient, $\mathrm{K}$ is kurtosis, and $\mathrm{D}$ is diffusivity.

DKI was imported into the software to obtain the final fitted images (ADC map, mean diffusivity [MD] map, and mean kurtosis [MK] map). The region of interest (ROI) of pre-chemotherapy was manually drawn on the axial plane encompassing the largest area of tumor on the b0 image while simultaneously avoiding encircling distortion artifacts and macroscopically visible necrotic, cystic, and hemorrhagic areas using the T2-weighted images (T2WI) and enhanced T1-weighted image (T1WI) as a guide (10). The largest area of the tumor was selected by two musculoskeletal radiologists, each with 20 years of diagnostic imaging experience, in consensus, and who were blinded to the patients' histologic information. The ROI was automatically matched on diffusivity maps and kurtosis maps. The pixelbased mean value was calculated, and ADC, MD, and MK values were obtained by the software. An example of an ROI is shown in Figure 1. For patients with good response after chemotherapy, the ROI was drawn on the same area that was initially used in the pre-chemotherapy MRI.

The tumor volume was independently calculated by two musculoskeletal radiologists. The maximal intramedullary extension of each lesion was measured from the coronal plane on T1WI, while the widths and depths were measured from the axial plane on fat-saturated T2WI. When these two reviewers found more than $10 \%$ discrepancy in tumor volume, the tumors were re-measured, and agreement was reached by consultation. The tumor volume was then calculated using the standard mathematical formula for an ellipsoid ( $0.52 \mathrm{x}$ length $\mathrm{x}$ width $\mathrm{x}$ depth) (18).

We defined change ratio $(\triangle X)$ in the $A D C, M D$, and $M K$ values and tumor volume using following formula (14):

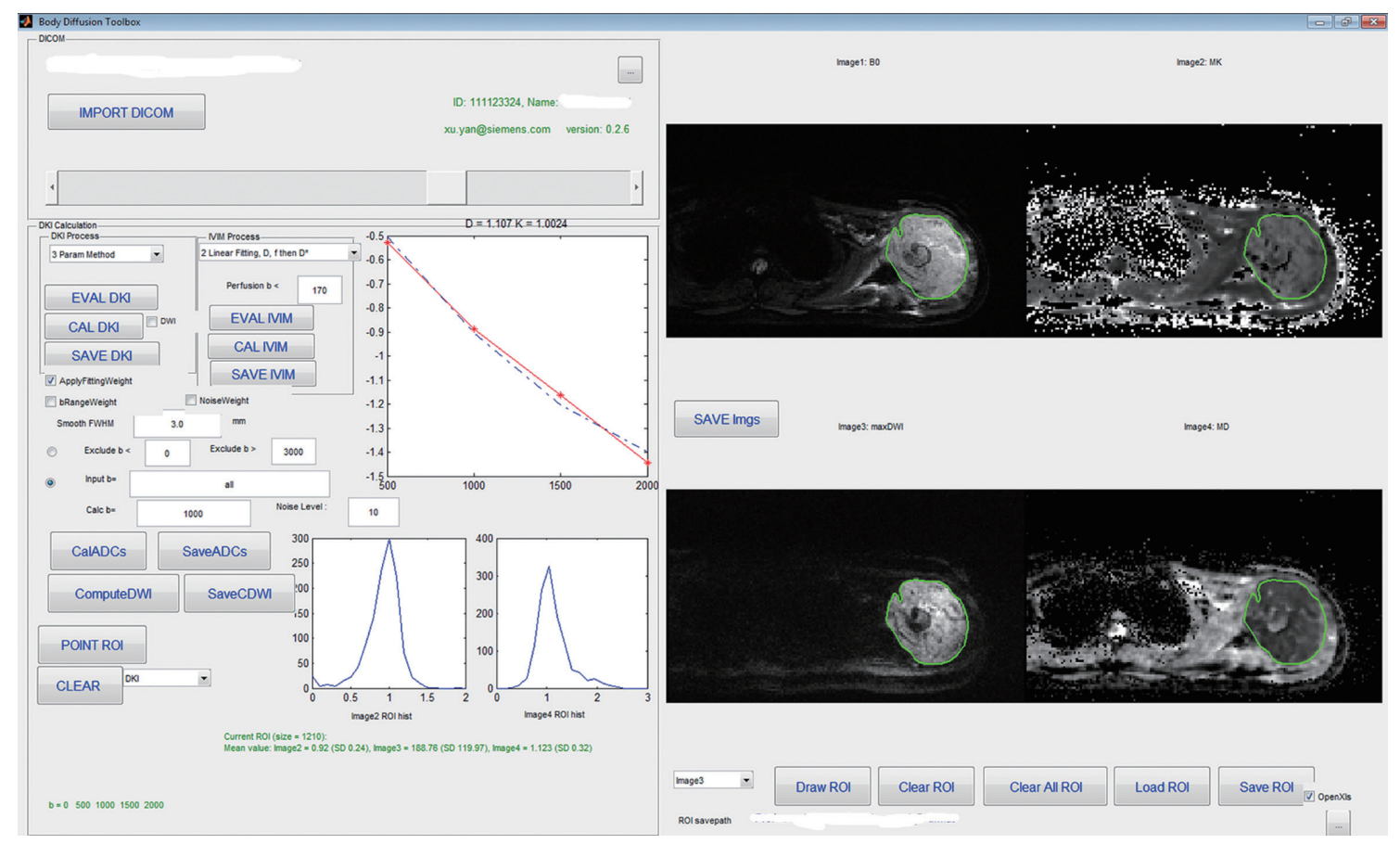

Fig. 1. Screenshot of placement of ROI, and DKI parameter maps using Body Diffusion Toolbox (Mathworks). DKI = diffusion kurtosis imaging, ROI = region of interest 
$\triangle A D C=\left(A D C_{\text {post }}-A D C_{\text {pre }}\right) / A D C_{\text {pre }} \Delta M D=\left(M D_{\text {post }}-M D_{\text {pre }}\right) /$ $M D_{\text {pre, }} \Delta M K=\left(M K_{\text {post }}-M K_{\text {pre }}\right) / M K_{\text {prer }} \Delta$ Volume $=\left(\right.$ Volume $_{\text {post }}-$ Volume $_{\text {pre }}$ ) / Volume pre

\section{Histopathologic Evaluation}

The resected specimen in at least two of the largest longitudinal sections and in various axial sections was

Table 1. Summary of Clinicopathological Characteristics of All Patients

\begin{tabular}{|c|c|c|c|}
\hline Characteristics & Good Responder $(n=12)$ & Poor Responder $(n=17)$ & $P$ \\
\hline Age & $14.5 \pm 6.1$ & $19.9 \pm 8.3$ & 0.259 \\
\hline \multicolumn{4}{|l|}{ Sex } \\
\hline Male & 8 & 12 & 0.822 \\
\hline Female & 4 & 5 & \\
\hline \multicolumn{4}{|l|}{ AJCC stage } \\
\hline II A & 3 & 1 & 0.470 \\
\hline II B & 7 & 12 & \\
\hline III & 1 & 1 & \\
\hline IV A & 1 & 3 & \\
\hline \multicolumn{4}{|l|}{ Pathologic subtypes } \\
\hline Osteoblastic & 11 & 15 & 0.678 \\
\hline Chondroblastic & 0 & 1 & \\
\hline Small cell & 1 & 1 & \\
\hline \multicolumn{4}{|l|}{ Tumor locations } \\
\hline Femur & 9 & 14 & 0.658 \\
\hline Tibia & 1 & 2 & \\
\hline Fibula & 1 & 0 & \\
\hline Humerus & 1 & 1 & \\
\hline
\end{tabular}

Student's $t$ test for age and chi-squared test for categorical variables. AJCC = American Joint Committee on Cancer

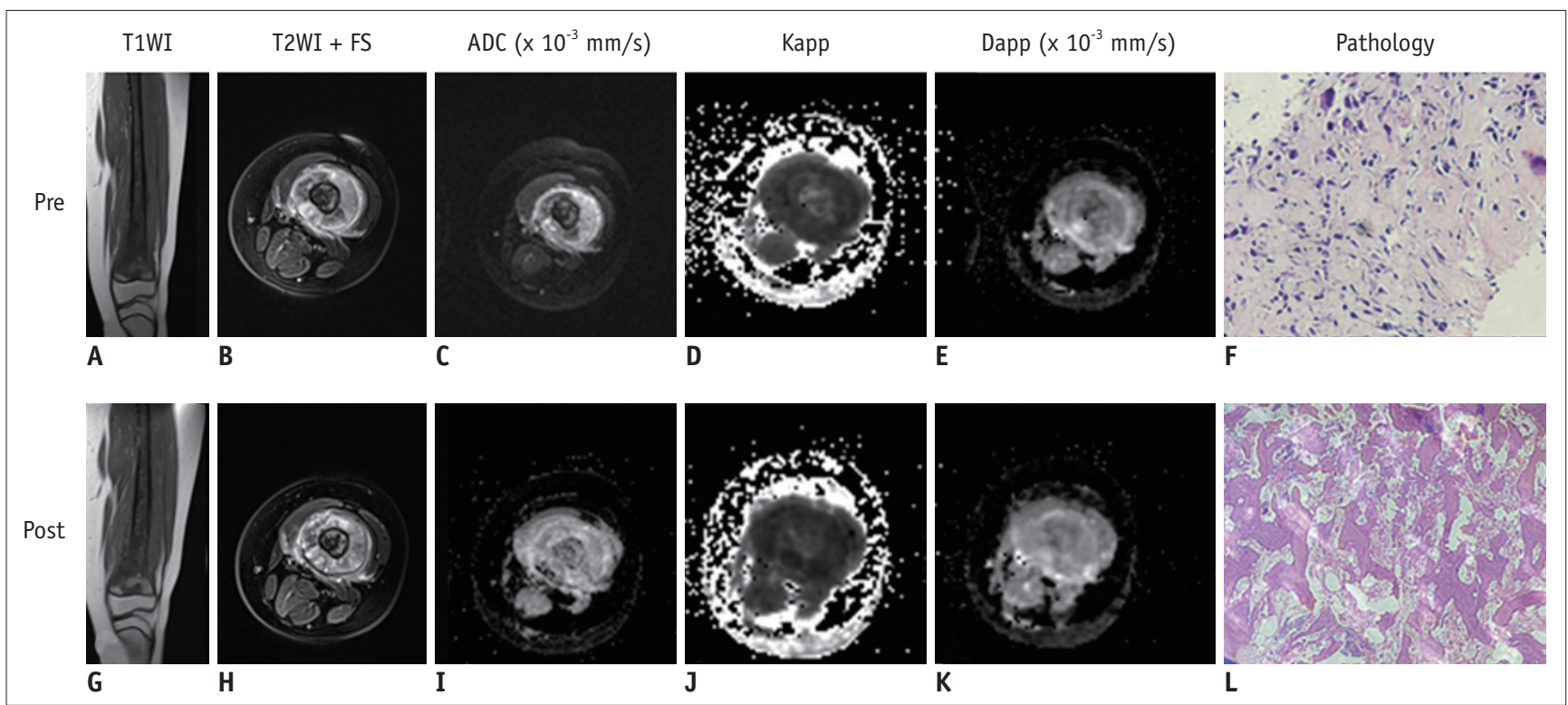

Fig. 2. Nine-year-old female with osteosarcoma in left femur and histopathology of poor responder.

Images before (A-F) and after (G-L) chemotherapy are shown. A. Coronal T1WI shows tumor located in metaphysis of left distal femur and extended to diaphysis. B. Axial T2WI with FS shows tumor heterogeneity. C. ADC map shows hyperintense tumor ( $\left.\mathrm{ADC}=0.97 \times 10^{-3} \mathrm{~mm}^{2} / \mathrm{s}\right)$. D. MK map shows mixed high and low signal intensity tumor $(M K=0.85)$. E. MD map shows mixed high and low signal intensity tumor $\left(M D=1.37 \times 10^{-3} \mathrm{~mm}^{2} / \mathrm{s}\right) . \mathrm{F}$ Photomicrography $(\mathrm{HE}, \mathrm{x} 200)$ confirms osteosarcoma. G. Coronal T2WI shows no significant change in intramedullary extension. H. Axial T2WI with FS magnetic resonance image shows changes in tumor structure compared to that of pre-chemotherapy. I. ADC value increased after chemotherapy (ADC $\left.=1.43 \times 10^{-3} \mathrm{~mm}^{2} / \mathrm{s}\right) . \mathrm{J}$. MK value decreased after chemotherapy $(\mathrm{MK}=0.61) . \mathrm{K}$. MD value also increased after chemotherapy $\left(\mathrm{MD}=1.87 \times 10^{-3} \mathrm{~mm}^{2} /\right.$ s). L. Photomicrography (HE, $\times 200)$ shows viable cellular areas. Necrosis was found in $60 \%$ of tumor. $A D C=$ apparent diffusion coefficient, Dapp $=$ diffusivity map, $\mathrm{FS}=$ fat saturation, $\mathrm{HE}=$ hematoxylin and eosin stain, Kapp = kurtosis map, MD = mean diffusivity, MK = mean kurtosis, T1WI = T1weighted imaging, $\mathrm{T} 2 \mathrm{WI}=\mathrm{T} 2$-weighted imaging 
examined by two experienced pathologists, and the tumor necrosis rate was determined. Tumor necrosis was graded as grade I for $0-49 \%$ necrosis, grade II for $50-$ $89 \%$ necrosis, grade III for $90-99 \%$ necrosis, and grade IV for $100 \%$ (19). Patients with grade III or IV ( $\geq 90 \%$ necrosis) were considered good responders, while those with grade I or II (<90\% necrosis) were considered poor responders.

\section{Statistical Analysis}

Statistical software packages (SPSS 16.0, SPSS Inc., Chicago, IL, USA; and MedCalc 14.0, MedCalc, Mariakerke, Belgium) were used to perform the statistical analyses. Two radiologists' measurements of $A D C, M K$, and MD values were analyzed using the interclass correlation coefficient (ICC). All data were first subjected to normal distribution using the Kolmogorov-Smirnov test. The differences between good responders and poor responders in various MRI parameters were compared using a two-tailed Student's $t$ test. The relationship between tumor necrosis and various MRI parameters was determined by Pearson's correlation analysis. The diagnostic performances of various MRI parameters were analyzed using receiver operating characteristics and the best threshold, sensitivity, and specificity were calculated. The area under the curve (AUC) of various MRI parameters were compared using the nonparametric statistical methods proposed by DeLong et al. (20). $P$ values less than 0.05 were considered to be statistically significant.

\section{RESULTS}

\section{Subject Characteristics}

The characteristics of all patients are summarized in Table 1. In the present study, 2 patients underwent amputation, while the others underwent segmental resection and prosthetic replacement. Based on tumor size, a mean of 24 (range, 14-41 pieces) resected specimens from each patient was used to assess tumor response to neoadjuvant chemotherapy. Seventeen patients showed poor response, and 12 patients showed good response (Figs. 2, 3). There was a similar age and sex composition between groups ( $p$ $=0.259$ and $p=0.822$, respectively), and no significant difference was observed in the tumor American Joint Committee on Cancer stages and pathologic subtypes ( $p=$ 0.470 and $p=0.678$, respectively).

\section{Reproducibility and Changes in MRI Parameters Based on} the Histologic Response

The ICC of the two radiologists' measurements were 0.891 (95\% confidence interval [CI]: 0.555, 0.977), 0.951 (95\%

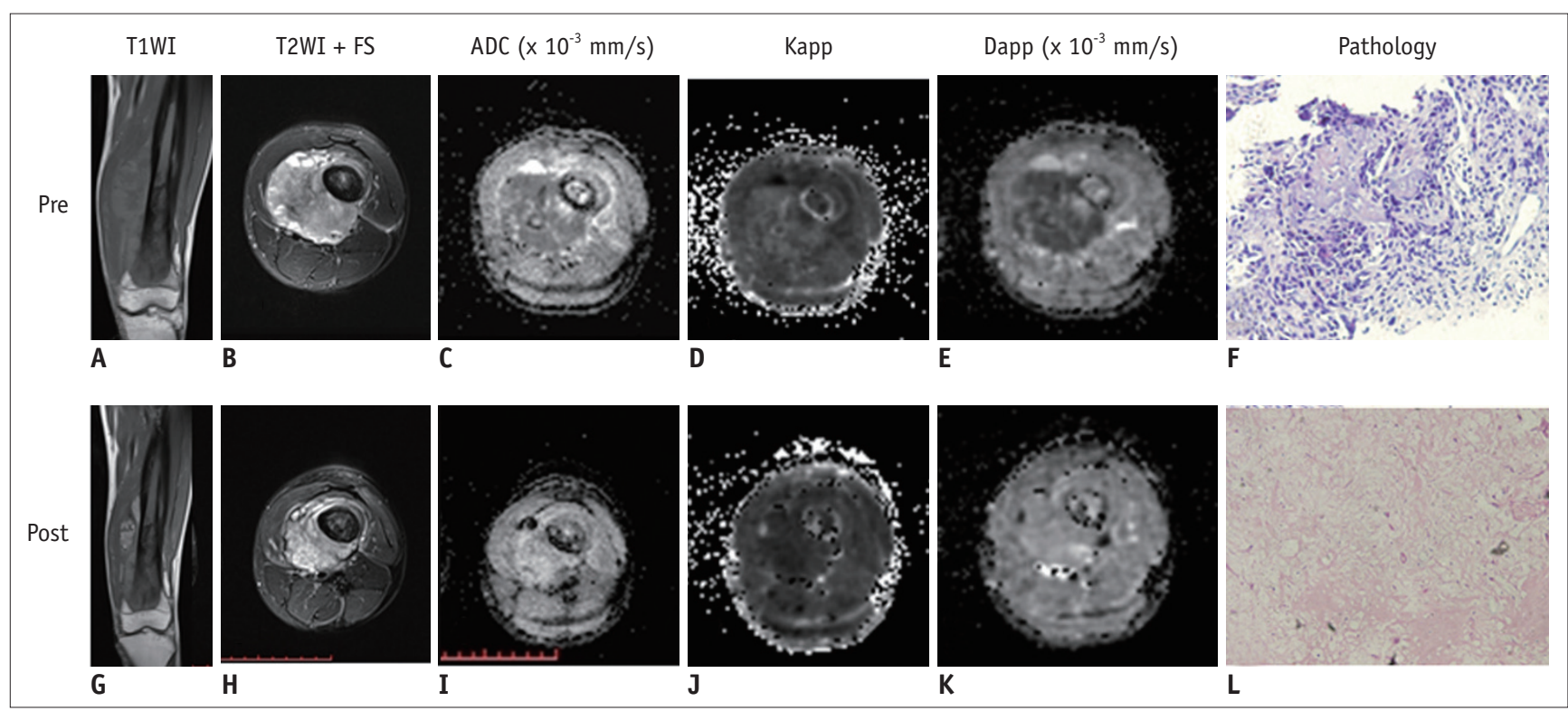

Fig. 3. Nineteen-year-old male with osteosarcoma and histopathology of good responses to neoadjuvant chemotherapy. Images before (A-F) and after (G-L) chemotherapy are shown. Compared to pre-chemotherapy, tumor size decreased on axial fat-saturated T2WI (B-H) whereas intramedullary extension changed little on coronal T1WI (A, G). ADC value significantly increased from $0.89 \times 10^{-3} \mathrm{~mm}^{2} / \mathrm{s}$ to $1.72 \times 10^{-3} \mathrm{~mm}^{2} / \mathrm{s}($ C, I), whereas MK values significantly decreased from 0.93 to 0.53 (D, J). MD value also significantly increased from $1.26 \times$ $10^{-3} \mathrm{~mm}^{2} / \mathrm{s}$ to $2.33 \times 10^{-3} \mathrm{~mm}^{2} / \mathrm{s}(\mathrm{E}, \mathrm{K})$. F. Photomicrography (HE, $\left.\times 200\right)$ showed osteosarcoma with CT-guided biopsy before chemotherapy. L. Photomicrography (HE, $\times 200$ ) depicts tumor necrosis rate of approximately $95 \%$ after chemotherapy. 
CI: $0.782,0.990)$, and 0.936 (95\% CI: $0.720-0.986)$ for $M D_{\text {pre, }} M K_{\text {pre, }}$ and $A D C_{\text {pre, }}$ respectively. The ICC for $M D_{\text {post }}$ $\mathrm{MK}_{\text {post }}$ and $\mathrm{ADC}_{\text {post }}$ were 0.890 (95\% CI: $\left.0.553,0.976\right), 0.826$ (95\% CI: $0.359,0.962)$, and 0.926 (95\% CI: $0.682,0.984$ ), respectively. There was good reproducibility for DKI-derived parameter analysis.

$M D_{\text {pre/post }}, M K_{\text {pre/post }}$, and $A D C_{\text {pre/post }}$ values and tumor volume $_{\text {pre/post }}$ exhibited normal distributions in both groups (Table 2). $M D_{\text {post }}$ and $A D C_{\text {post }}$ values were significantly higher in good responders ( $p<0.001$ for both), and $\mathrm{MK}_{\text {post }}$ values were lower in poor responders $(p=0.042)$. MD and ADC increased by $55.5 \pm 26.1 \%$ and $47.2 \pm 43.2 \%$, respectively, while MK decreased by $-20.6 \pm 26.8 \%$ in good responders relative to the baseline. The $\triangle M D$ and $\triangle A D C$ in good responders were significantly higher than those in poor responders ( $p<0.001$ and $p=0.01$, respectively). However, no significant difference was observed in $\triangle \mathrm{MK}(p=0.092)$. Tumor volume post $_{\text {and }} \Delta$ tumor volume between groups also did not reach statistical significance $(p=0.706$ and $p=$ 0.068 , respectively) (Fig. 4 , Table 2 ).

\section{Relationship between Tumor Necrosis and Imaging}

Indexes, and Diagnostic Performance Analyses

The correlation between tumor necrosis rate and MRI indexes are summarized in Table 3. $M D_{\text {post }}$ and $\triangle M D$ showed high correlation ( $r=0.669$ and $r=0.622$, respectively) with tumor necrosis rate (Fig. 5). $A D C_{\text {post }}$ and $\triangle A D C$ were positively correlated with tumor necrosis rate $(r=0.462$ $[p=0.012]$ and $r=0.447[p=0.015]$, respectively), while $M K_{\text {post }}$ and $\triangle M K$ showed no significant correlation $(p=0.121$ and $p=0.171$, respectively).

Table 4 shows various MRI parameters to access good response to chemotherapy. $M D_{\text {post }}$ resulted in the highest AUC of 0.91 (95\% CI: $0.753,0.986 . p<0.001$ ), with the optimal cutoff at $1.61 \times 10^{-3} \mathrm{~mm}^{2} / \mathrm{s}$ (sensitivity, $100 \%$; specificity, $70.6 \%)$. For comparison of $\mathrm{MD}_{\text {post }}, A D C_{\text {post }}$ and $\mathrm{MK}_{\text {post }}, \mathrm{MD}_{\text {post }}$ had higher diagnostic performance than $A D C_{\text {post }}$ $(p=0.037)$ and $\mathrm{MK}_{\text {post }}(p=0.011) . \mathrm{ADC}_{\text {post }}$ and $\mathrm{MK}_{\text {post }}$ had a similar diagnostic performance $(p=0.058)$. For comparison of $\triangle M D, \triangle A D C$, and $\triangle M K, \triangle M D$ presented the highest AUC of 0.92 (95\% CI: $0.759,0.988 . p<0.001$ ), with the optimal cut off at $13.5 \%$ (sensitivity, $100 \%$; specificity, $70.6 \%$ ). Similarly, $\triangle M D$ showed higher diagnostic performance than $\triangle \operatorname{ADC}(p=0.033)$ and $\triangle \mathrm{MK}(p=0.037)$. No significant difference was observed in the diagnostic performance of $\triangle \mathrm{ADC}$ and $\triangle \mathrm{MK}(p=0.434)$ (Fig. 6).

\section{DISCUSSION}

Our initial findings showed that MD may potentially serve as an optimal surrogate imaging biomarker for histological tumor necrosis in patients with osteosarcoma.

Until now, there has been no agreement on the relationship between histological tumor necrosis and $A D C$ values. Uhl et al. (21) and Hayashida et al. (14) confirmed the ability of DWI to distinguish good and poor responders based on a short series. However, with a larger series of osteosarcoma patients, $0 \mathrm{ka}$ et al. (13) and Bajpai et al. (12) found no significant difference between good and poor responders in terms of $A D C$ measured at the end

Table 2. Comparison of Various MRI Parameters between Good Responders and Poor Responders

\begin{tabular}{|c|c|c|c|c|c|c|}
\hline \multirow{2}{*}{ Variable } & \multicolumn{2}{|c|}{ Pre-Treatment } & \multicolumn{2}{|c|}{ Post-Treatment } & \multicolumn{2}{|c|}{ Change Ratio (\%) } \\
\hline & Good Responder & Poor Responder & Good Responder & Poor Responder & Good Responder & Poor Responder \\
\hline $\mathrm{MD}\left(\times 10^{-3} \mathrm{~mm}^{2} / \mathrm{s}\right)$ & $1.29 \pm 0.23$ & $1.29 \pm 0.21$ & $1.97 \pm 0.21$ & $1.39 \pm 0.39$ & $55.5 \pm 26.1$ & $7.0 \pm 25.2$ \\
\hline$p$ & 0.982 & & $<0.001$ & & $<0.001$ & \\
\hline$p^{*}$ & 0.980 & 0.841 & 0.975 & 0.731 & 0.887 & 0.990 \\
\hline MK & $0.92 \pm 0.25$ & $0.91 \pm 0.14$ & $0.68 \pm 0.14$ & $0.86 \pm 0.26$ & $-20.6 \pm 26.8$ & $-5.2 \pm 20.8$ \\
\hline$p$ & 0.857 & & 0.042 & & 0.092 & \\
\hline$p^{*}$ & 0.178 & 0.999 & 0.964 & 0.538 & 0.941 & 0.860 \\
\hline $\mathrm{ADC}\left(\times 10^{-3} \mathrm{~mm}^{2} / \mathrm{s}\right)$ & $0.98 \pm 0.20$ & $0.91 \pm 0.17$ & $1.39 \pm 0.24$ & $1.00 \pm 0.31$ & $47.2 \pm 43.2$ & $10.1 \pm 28.8$ \\
\hline$p$ & 0.309 & & 0.001 & & 0.010 & \\
\hline$p^{*}$ & 0.999 & 0.714 & 0.538 & 0.674 & 0.871 & 0.848 \\
\hline Tumor volume & $425.63 \pm 390.94$ & $316.23 \pm 151.46$ & $379.59 \pm 372.49$ & $339.05 \pm 196.67$ & $-0.1 \pm 0.1$ & $0.1 \pm 0.4$ \\
\hline$p$ & 0.373 & & 0.706 & & 0.068 & \\
\hline$p^{*}$ & 0.538 & 0.945 & 0.257 & 0.991 & 0.892 & 0.965 \\
\hline
\end{tabular}

Data are mean \pm standard deviation. Tumor volume is given in $\mathrm{cm}^{3} .{ }^{*} p$ value for normality test. $A D C=$ apparent diffusion coefficient, $M D=$ mean diffusivity, $M K=$ mean kurtosis 

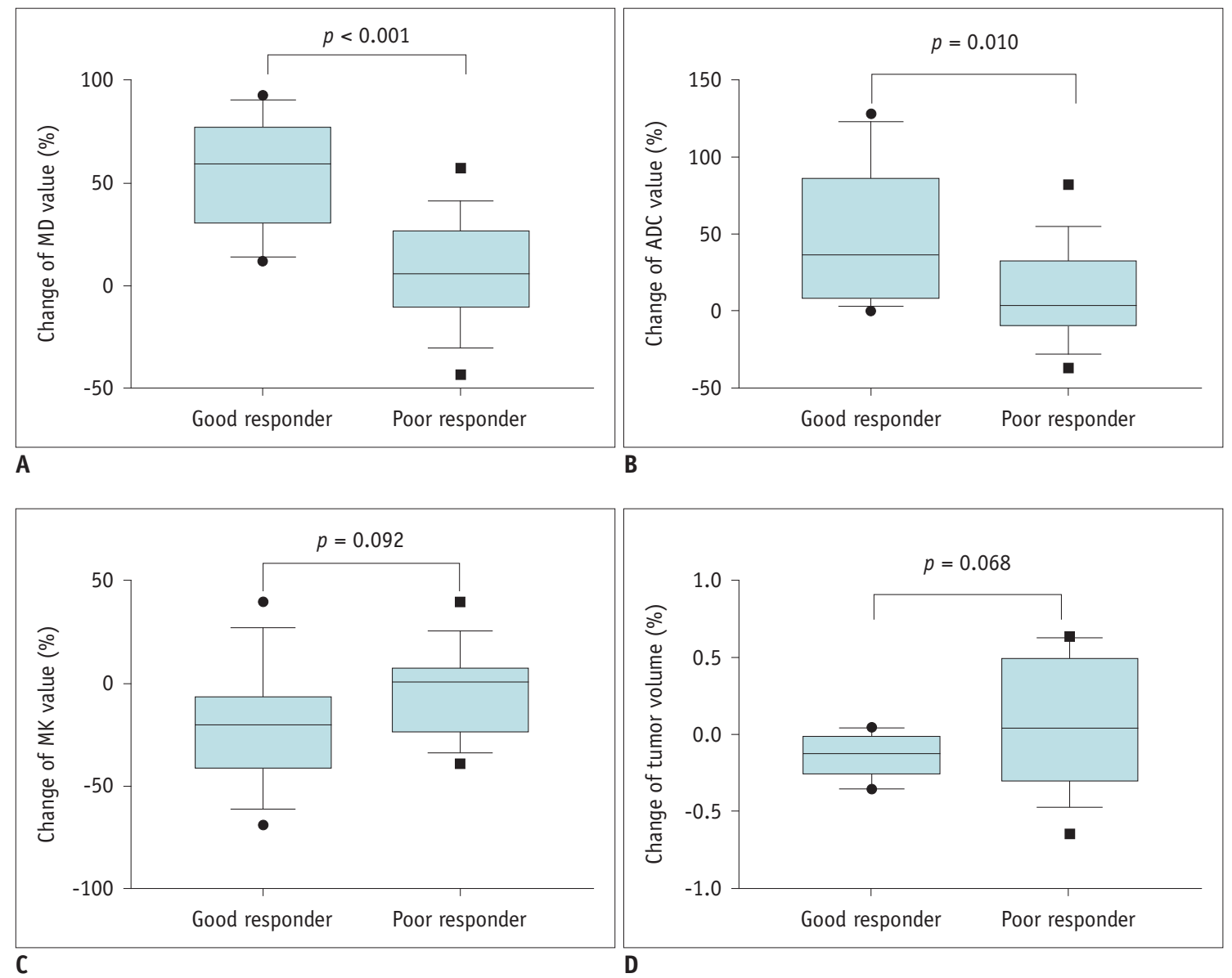

Fig. 4. Box plots show $\triangle M D_{10 t h}, \triangle A D C_{10 t h}, \triangle M K_{10 t h}$ and $\triangle$ tumor volume ${ }_{10 t h}$ in good responders and poor responders.

A. Compared to poor responders, there was statistically significant difference in $\triangle M D$ of good responders $(p<0.001)$. B. $\triangle A D C$ was significantly higher in good responders than in poor responders $(p=0.010)$. C. No significant difference in $\triangle M K$ was observed between good responders and poor responders $(p=0.092)$. D. There was also no significant difference in $\Delta$ tumor volume $(p=0.068) . \Delta \mathrm{X}=$ change ratio

of the chemotherapy. In our present study, the results showed a significant difference in $M D_{\text {post }}$ and $\triangle M D$ between patients with good and poor responses. Compared to $M D_{\text {post }}$ and $\triangle M D$ in poor responders, those in good responders were significantly increased, which may be due to postchemotherapeutic changes, such as cell and intracellular membrane injury or cell death, or a decrease in tumor cell density could cause an expansion of the extracellular space and lead to free diffusion of extracellular water.

Compared to $\triangle A D C, \triangle M D$ showed higher diagnostic performance and higher correlation with tumor necrosis. These may be explained as follows: first, the DWI model supposed that water diffusion in tissue was normally distributed and that signal intensity presented linear decay as the $b$ values increased, whereas the DKI model analyzed the water diffusion as a non-Gaussian phenomenon by a polynomial model. MD is the corrected diffusion coefficient accounting for non-Gaussian behavior, which could better
Table 3. Correlation between Tumor Necrosis Rate and Diffusion-Weighted MRI Parameters

\begin{tabular}{lrr}
\hline \multicolumn{1}{c}{ Variable } & $r$ & \multicolumn{1}{c}{$P$} \\
\hline $\mathrm{MD}_{\text {pre }}\left(\times 10^{-3} \mathrm{~mm}^{2} / \mathrm{s}\right)$ & 0.171 & 0.375 \\
$\mathrm{MK}_{\text {pre }}$ & -0.197 & 0.306 \\
$\mathrm{ADC}_{\text {pre }}\left(\times 10^{-3} \mathrm{~mm}^{2} / \mathrm{s}\right)$ & 0.283 & 0.137 \\
Tumor volume $_{\text {pre }}\left(\mathrm{cm}^{3}\right)$ & -0.119 & 0.540 \\
$\mathrm{MD}_{\text {post }}\left(\times 10^{-3} \mathrm{~mm}^{2} / \mathrm{s}\right)^{*}$ & 0.669 & $<0.001$ \\
$M K_{\text {post }}$ & -0.294 & 0.121 \\
$A D C_{\text {post }}\left(\times 10^{-3} \mathrm{~mm}^{2} / \mathrm{s}\right)$ & 0.462 & 0.012 \\
Tumor volume & -0.193 & 0.316 \\
$\Delta M D^{*}$ & 0.622 & $<0.001$ \\
$\triangle M K$ & -0.261 & 0.171 \\
$\triangle A D C$ & 0.447 & 0.015 \\
$\Delta$ Tumor volume & -0.197 & 0.306 \\
\hline
\end{tabular}

*High correlation $(r>0.6)$ is demonstrated at 0.005 level (2-tailed). $\Delta \mathrm{X}=$ change ratio

reflect water diffusivity. Second, the ADC measurement only reflects the degree of water molecular diffusion hindered in extracellular space, and easily influenced by tissue 


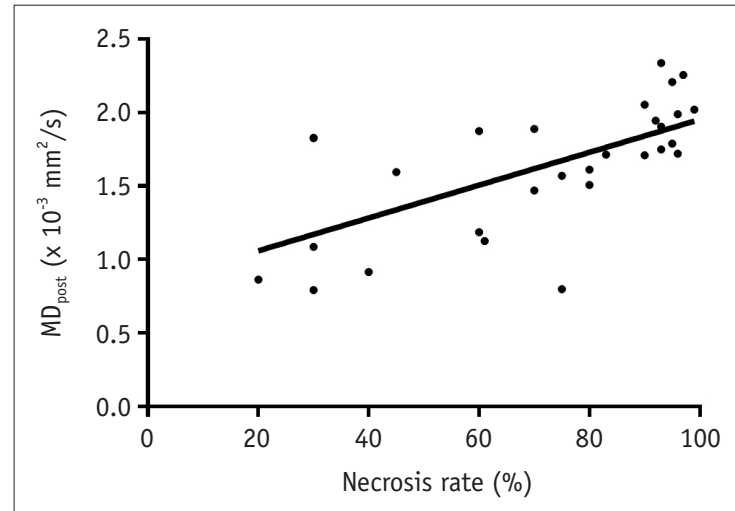

A

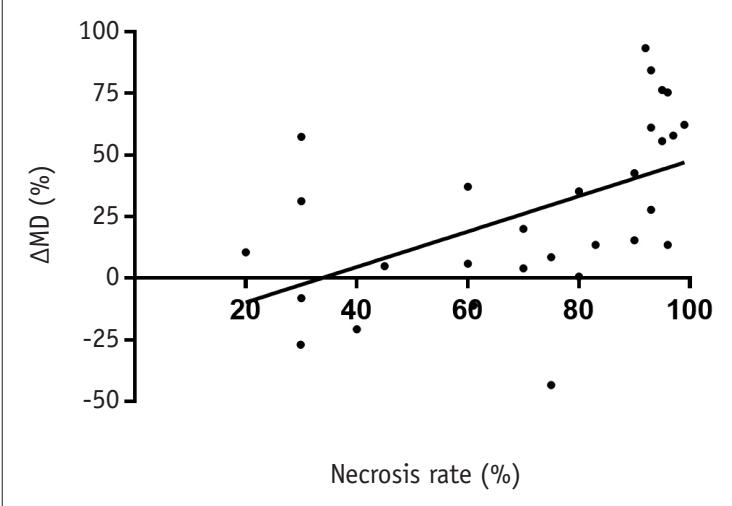

B

Fig. 5. Scatterplot showing relationship between tumor necrosis rate and $M D_{\text {post }}$ and $\triangle M D$.

A. $M D_{\text {post }}$ was positively correlated with necrosis rate $(r=0.669, p<0.001)$. B. $\triangle M D$ also showed positive correlation with necrosis rate $(r=0.622$, $p<0.001)$.

Table 4. Diagnostic Performance for Diffusion-Weighted MRI Indexes in Assessing Good Response

\begin{tabular}{|c|c|c|c|c|c|c|c|}
\hline Variables & Cutoff Value & AUC & Sensitivity (\%) & Specificity (\%) & $\mathrm{LR+}$ & LR- & $P$ \\
\hline$M D_{\text {pre }}$ & $\leq 1.08$ & $0.52(0.327-0.708)$ & $25.0(5.5-57.2)$ & $88.2(63.6-98.5)$ & 2.12 & 0.85 & 0.86 \\
\hline$M K_{\text {pre }}$ & $\leq 0.93$ & $0.56(0.368-0.746)$ & $83.3(51.6-97.9)$ & $47.1(23.0-72.2)$ & 1.57 & 0.35 & 0.58 \\
\hline$A D C_{\text {pre }}$ & $>0.78$ & $0.61(0.420-0.791)$ & $91.7(61.5-99.8)$ & $35.3(14.2-61.7)$ & 1.42 & 0.24 & 0.28 \\
\hline Tumor volume $_{\text {pre }}\left(\mathrm{cm}^{3}\right)$ & $>393.92$ & $0.52(0.327-0.708)$ & $41.7(15.2-72.3)$ & $82.4(56.6-96.2)$ & 2.36 & 0.71 & 0.87 \\
\hline$M D_{\text {post }}$ * & $>1.61$ & $0.91(0.753-0.986)$ & $100.0(73.5-100.0)$ & $70.6(44.0-89.7)$ & 3.40 & 0.00 & $<0.001$ \\
\hline$M K_{\text {post }}{ }^{*}$ & $\leq 0.80$ & $0.72(0.524-0.870)$ & $91.7(61.5-99.8)$ & $47.1(23.0-72.2)$ & 1.73 & 0.18 & 0.02 \\
\hline$A D C_{\text {post }}{ }^{*}$ & $>1.16$ & $0.80(0.621-0.930)$ & $91.7(61.5-99.8)$ & $70.6(44.0-89.7)$ & 3.12 & 0.12 & $<0.001$ \\
\hline Tumor volume $_{\text {post }}\left(\mathrm{cm}^{3}\right)$ & $\leq 255.93$ & $0.54(0.345-0.725)$ & $58.3(27.7-84.8)$ & $58.8(32.9-81.6)$ & 1.42 & 0.71 & 0.73 \\
\hline$\Delta M D^{*}$ & $>13.50$ & $0.92(0.759-0.988)$ & $100.0(73.5-100.0)$ & $70.6(44.0-89.7)$ & 3.40 & 0.00 & $<0.001$ \\
\hline$\Delta M K^{*}$ & $\leq-1.36$ & $0.72(0.519-0.867)$ & $91.7(61.5-99.8)$ & $52.9(27.8-77.0)$ & 1.95 & 0.16 & 0.034 \\
\hline$\triangle A D C^{*}$ & $>6.30$ & $0.78(0.587-0.911)$ & $91.7(61.5-99.8)$ & $58.8(32.9-81.6)$ & 2.23 & 0.14 & 0.001 \\
\hline$\Delta$ Tumor volume & $\leq 0.01$ & $0.64(0.444-0.810)$ & $91.7(61.5-99.8)$ & $58.8(32.9-81.6)$ & 2.23 & 0.14 & 0.19 \\
\hline
\end{tabular}

Data in brackets are $95 \%$ confidence intervals. MD and ADC are given in $\mathrm{mm}^{2} / \mathrm{s} \times 10^{-3} .{ }^{*} p<0.05 . \mathrm{AUC}=$ area under curve, $\mathrm{LR}=$ likelihood ratio

architectural properties such as vessel and extracellular space tortuosity (22). In contrast, MD could reflect water's molecular heterogeneous properties at the nanoscopic level including its polar nature and 3-dimensional array (23). Third, ADC could not specifically reflect cellular density on water motion; it was influenced by the presence of a greater concentration of macromolecules and increased viscosity (24).

In contrast to $\mathrm{MD}_{\text {post }}, \mathrm{MK}_{\text {post }}$ in poor responders was higher than that in good responders. Studies showed that higher MK values indicated increased irregularity and heterogeneity of tissue microstructure as well as the amount of interface of cellular tissues (25). In poor responders, tumors were insensitive or resistant to chemotherapy; the toxicity of anti-tumor chemotherapeutics may induce mitochondrial and organelle swelling, increase the nuclear-cytoplasmic ratio of tumor cells, or induce mitosis of tumor cells and proliferate and form new colonies. Moreover, after chemotherapy, slight necrosis, hemorrhage, granulation tissue formation, and fibrosis further increase structural complexity (26). Surprisingly, no significant difference was observed in $\triangle M K$ between good and poor responders. On one hand, this may be related to the small number of subjects. On the other hand, in some areas of chemotherapy-induced viscous or turbid fluid, MD was reduced while MK was not increased. Moreover, compared to $A D C_{\text {post }}, \triangle A D C$, and $M K_{\text {post }} \triangle M K$ had lower specificity, which may be related to the intrinsic nature of osteosarcoma. Compared with other tissues, bone tumors may exhibit faster signal decays as $b$ values increase (27). Furthermore, in good responders, there was extensive inflammatory cell infiltration, interstitial fibrosis, and granulation tissue in the bone marrow cavity with the 


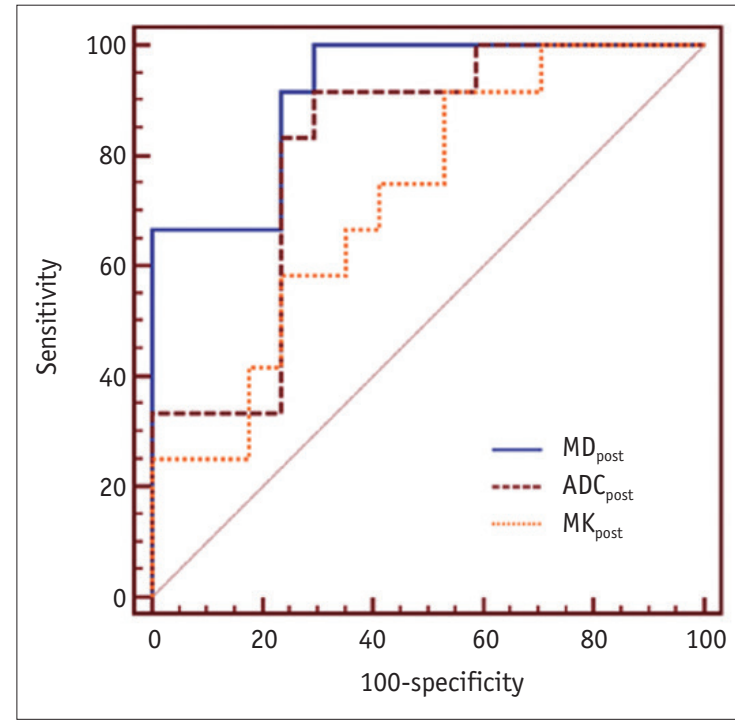

A

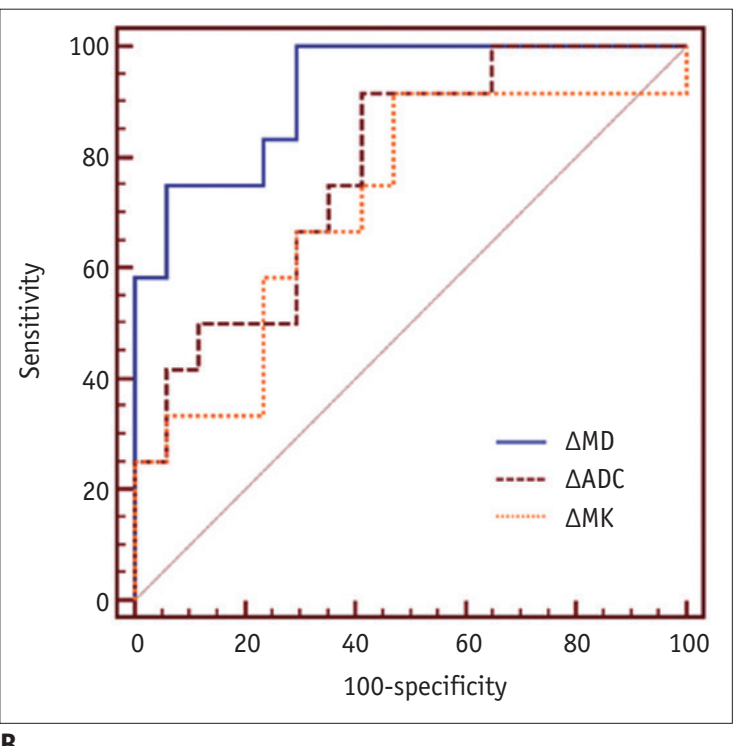

B

Fig. 6. A. ROC curves showing diagnostic performance of $M D_{\text {post }} A D C_{\text {post }}$ and $M K_{\text {post }}$ in assessing good response to neoadjuvant chemotherapy. $M D_{\text {post }}$ resulted in highest AUC of $0.91(95 \% \mathrm{CI}: 0.753,0.986 . p<0.001)$, and $\mathrm{MD}_{\text {post }}$ had higher diagnostic performance than $A D C_{\text {post }}(p=0.037)$ and $\mathrm{MK}_{\text {post }}(p=0.011)$. B. ROC curves showing diagnostic performance of $\triangle M D, \triangle A D C$, and $\triangle M K . \triangle M D$ presented highest AUC (0.92, 95\% CI: 0.759 , $0.988, p<0.001$ ), with optimal cutoff value at $13.5 \%$ (sensitivity, $100 \%$; specificity, $70.6 \%$ ), and $\triangle M D$ showed higher diagnostic performance than $\triangle \mathrm{ADC}(p=0.033)$ and $\triangle \mathrm{MK}(p=0.037) . \mathrm{AUC}=$ area under curve, $\mathrm{CI}=$ confidence interval, $\mathrm{ROC}=$ receiver operating characteristic

tumor necrosis. This may partially explain our findings.

Our study also showed no significant difference in the change in tumor volumes between good responders and poor responders. Although the change in tumor size remains the standard modality for monitoring response to treatment in current international therapy optimization trials, our findings confirmed that this is not sufficient to monitor tumor regression. This is mainly because chemotherapyinduced response in intraosseous or mineralized tumor partitions can leave imprints that lead to only a little change in tumor volumes, especially in tumors with little or no soft tissue component (6). Moreover, in some patients with good response, tumor volume was stable or even larger than pre-chemotherapy due to cystic degeneration (12).

There are several potential limitations in our study. First, the number of subjects was small, which may result in biased conclusions. Second, the follow-up period was relatively short, and we could not analyze the relationship between survival rate and DKI parameters. Third, DKI required a relatively long acquisition time, which may have increased motion artifacts. Fourth, irregular tumor shapes may not fit well into an ellipsoid formula, which may cause under- or over-estimation of tumor volume. Fifth, CT-guided biopsy pre-chemotherapy may slightly affect tumor composition and measurement in MD, MK, and ADC values. Sixth, the minimal $b$ value of 0 for DKI was used in our study, which might have increased the intravoxel incoherent motion effects. Finally, early mid-therapy and the time course of DWI kinetic parameter changes should be explored further.

In summary, MD is a promising biomarker for monitoring tumor response to preoperative chemotherapy in patients with osteosarcoma, and $M D_{\text {post }}$ and $\triangle M D$ were found to serve as potentially optimal surrogate imaging biomarkers for histological tumor necrosis, which will contribute to decisions regarding individual surgical planning, additional treatment, and follow-up strategies.

\section{Conflicts of Interest}

The authors have no potential conflicts of interest to disclose.

\section{Acknowledgments}

The manuscript had been edited by American Journal Experts.

ORCID iDs

Weiwu Yao

https://orcid.org/0000-0002-1536-9324

Chenglei Liu

https://orcid.org/0000-0002-5671-8236

Yan Xi

https://orcid.org/0000-0001-6143-3107 
Mei Li

$$
\begin{aligned}
& \text { https://orcid.org/0000-0003-1674-9270 } \\
& \text { Qiong Jiao } \\
& \text { https://orcid.org/0000-0002-7782-7917 } \\
& \text { Huizhen Zhang } \\
& \text { https://orcid.org/0000-0002-8760-4178 } \\
& \text { Qingcheng Yang } \\
& \text { https://orcid.org/0000-0002-8948-4296 }
\end{aligned}
$$

\section{REFERENCES}

1. de Baere T, Vanel D, Shapeero LG, Charpentier A, Terrier P, di Paola M. Osteosarcoma after chemotherapy: evaluation with contrast material-enhanced subtraction MR imaging. Radiology 1992;185:587-592

2. Bielack SS, Hecker-Nolting S, Blattmann C, Kager L. Advances in the management of osteosarcoma. F1000Res 2016;5:2767

3. Davis AM, Bell RS, Goodwin PJ. Prognostic factors in osteosarcoma: a critical review. J Clin Oncol 1994;12:423-431

4. Denecke T, Hundsdorfer P, Misch D, Steffen IG, Schonberger $S$, Furth $C$, et al. Assessment of histological response of paediatric bone sarcomas using FDG PET in comparison to morphological volume measurement and standardized MRI parameters. Eur J Nucl Med Mol Imaging 2010;37:1842-1853

5. Kubo T, Furuta T, Johan MP, Adachi N, Ochi M. Percent slope analysis of dynamic magnetic resonance imaging for assessment of chemotherapy response of osteosarcoma or Ewing sarcoma: systematic review and meta-analysis. Skeletal Radiol 2016;45:1235-1242

6. Brisse H, Ollivier L, Edeline V, Pacquement H, Michon J, Glorion $\mathrm{C}$, et al. Imaging of malignant tumours of the long bones in children: monitoring response to neoadjuvant chemotherapy and preoperative assessment. Pediatr Radiol 2004;34:595-605

7. Wakabayashi H, Saito J, Taki J, Hashimoto N, Tsuchiya H, Gabata T, et al. Triple-phase contrast-enhanced MRI for the prediction of preoperative chemotherapeutic effect in patients with osteosarcoma: comparison with (99m)Tc-MIBI scintigraphy. Skeletal Radiol 2016;45:87-95

8. Lang P, Wendland MF, Saeed M, Gindele A, Rosenau W, Mathur $A$, et al. Osteogenic sarcoma: noninvasive in vivo assessment of tumor necrosis with diffusion-weighted MR imaging. Radiology 1998;206:227-235

9. Wang CS, Du LJ, Si MJ, Yin QH, Chen L, Shu M, et al. Noninvasive assessment of response to neoadjuvant chemotherapy in osteosarcoma of long bones with diffusionweighted imaging: an initial in vivo study. PLoS One 2013;8:e72679

10. Byun BH, Kong CB, Lim I, Choi CW, Song WS, Cho WH, et al. Combination of 18F-FDG PET/CT and diffusion-weighted MR imaging as a predictor of histologic response to neoadjuvant chemotherapy: preliminary results in osteosarcoma. J Nucl
Med 2013;54:1053-1059

11. Baunin C, Schmidt G, Baumstarck K, Bouvier C, Gentet JC, Aschero $A$, et al. Value of diffusion-weighted images in differentiating mid-course responders to chemotherapy for osteosarcoma compared to the histological response: preliminary results. Skeletal Radiol 2012;41:1141-1149

12. Bajpai J, Gamnagatti S, Kumar R, Sreenivas V, Sharma MC, Khan SA, et al. Role of MRI in osteosarcoma for evaluation and prediction of chemotherapy response: correlation with histological necrosis. Pediatr Radiol 2011;41:441-450

13. Oka K, Yakushiji T, Sato H, Hirai T, Yamashita Y, Mizuta H. The value of diffusion-weighted imaging for monitoring the chemotherapeutic response of osteosarcoma: a comparison between average apparent diffusion coefficient and minimum apparent diffusion coefficient. Skeletal Radiol 2010;39:141146

14. Hayashida Y, Yakushiji T, Awai K, Katahira K, Nakayama Y, Shimomura 0, et al. Monitoring therapeutic responses of primary bone tumors by diffusion-weighted image: initial results. Eur Radiol 2006;16:2637-2643

15. Yu J, Xu Q, Song JC, Li Y, Dai X, Huang DY, et al. The value of diffusion kurtosis magnetic resonance imaging for assessing treatment response of neoadjuvant chemoradiotherapy in locally advanced rectal cancer. Eur Radiol 2017;27:1848-1857

16. Wang D, Li YH, Fu J, Wang H. Diffusion kurtosis imaging study on temporal lobe after nasopharyngeal carcinoma radiotherapy. Brain Res 2016;1648:387-393

17. Zhu L, Pan Z, Ma Q, Yang W, Shi H, Fu C, et al. Diffusion kurtosis imaging study of rectal adenocarcinoma associated with histopathologic prognostic factors: preliminary findings. Radiology 2017;284:66-76

18. Bieling P, Rehan N, Winkler P, Helmke K, Maas R, Fuchs $\mathrm{N}$, et al. Tumor size and prognosis in aggressively treated osteosarcoma. J Clin Oncol 1996;14:848-858

19. Rosen G, Caparros B, Huvos AG, Kosloff C, Nirenberg A, Cacavio A, et al. Preoperative chemotherapy for osteogenic sarcoma: selection of postoperative adjuvant chemotherapy based on the response of the primary tumor to preoperative chemotherapy. Cancer 1982;49:1221-1230

20. DeLong ER, DeLong DM, Clarke-Pearson DL. Comparing the areas under two or more correlated receiver operating characteristic curves: a nonparametric approach. Biometrics 1988;44:837-845

21. Uhl M, Saueressig U, Koehler G, Kontny U, Niemeyer C, Reichardt W, et al. Evaluation of tumour necrosis during chemotherapy with diffusion-weighted MR imaging: preliminary results in osteosarcomas. Pediatr Radiol 2006;36:1306-1311

22. Rosenkrantz AB, Padhani AR, Chenevert TL, Koh DM, De Keyzer F, Taouli B, et al. Body diffusion kurtosis imaging: basic principles, applications, and considerations for clinical practice. J Magn Reson Imaging 2015;42:1190-1202

23. Le Bihan D. The 'wet mind': water and functional neuroimaging. Phys Med Biol 2007;52:R57-R90 
24. Jensen JH, Helpern JA, Ramani A, Lu H, Kaczynski K. Diffusional kurtosis imaging: the quantification of nonGaussian water diffusion by means of magnetic resonance imaging. Magn Reson Med 2005;53:1432-1440

25. Jensen JH, Helpern JA. MRI quantification of nonGaussian water diffusion by kurtosis analysis. NMR Biomed 2010;23:698-710
26. Pan G, Raymond AK, Carrasco CH, Wallace S, Kim EE, Shirkhoda A, et al. Osteosarcoma: MR imaging after preoperative chemotherapy. Radiology 1990;174:517-526

27. Subhawong TK, Jacobs MA, Fayad LM. Diffusion-weighted MR imaging for characterizing musculoskeletal lesions.

Radiographics 2014;34:1163-1177 